\title{
Direct-acting Antiviral Therapy Improves the Outcome of Chronic Hepatitis C/intermediate-stage B Hepatocellular Carcinoma Patients
}

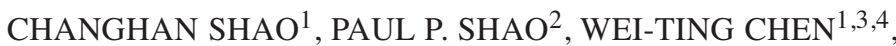 \\ CHENG-CHUN LIN ${ }^{1,3,4}$, WEY-RAN LIN ${ }^{1,3,4}$ and CHAU-TING YEH ${ }^{1,3,4}$ \\ ${ }^{1}$ Department of Gastroenterology and Hepatology, Linkou Medical Center, \\ Chang Gung Memorial Hospital, Taoyuan City, Taiwan, R.O.C., \\ ${ }^{2}$ David Geffen School of Medicine at University of California, Los Angeles, CA, U.S.A.; \\ ${ }^{3}$ Liver Research Center, Chang Gung Memorial Hospital, Taoyuan City, Taiwan, R.O.C.; \\ ${ }^{4}$ Chang Gung University College of Medicine, Taoyuan City, Taiwan, R.O.C.
}

\begin{abstract}
Background/Aim: The benefit of direct-acting antiviral therapy (DAA) in chronic hepatitis $C(\mathrm{CHC})$ infected patients who received curative treatment for early-stage hepatocellular carcinoma (HCC) is well known, but is unclear for intermediate stage HCC. Patients and Methods: CHC patients with Barcelona Clinic Liver Cancer (BCLC) stage B $H C C$ receiving chemoembolization were identified. Univariate, multivariate analyses, and Kaplan-Meier curve were used to identify factors associated with survival outcomes. Results: Among 113 included patients, the median survival of DAA treated group $(n=14)$ and non-treated group $(n=99)$ were 40.1 months and 22.9 months, respectively. Multivariate analysis showed that Eastern Cooperative Oncology Group (ECOG) score, DAA, and serum albumin were key independent factors associated with overall survival. Moreover, the time-tocomplete remission (TTCR) was improved in the DAA treated group. Conclusion: ECOG, DAA, and serum albumin were prognostic factors for CHC/intermediate-stage HCC patients. DAA was also a beneficial factor for TTCR.
\end{abstract}

This article is freely accessible online.

Correspondence to: Wey-Ran Lin, MD, Ph.D., AGAF, Department of Gastroenterology and Hepatology, Linkou Medical Center, Chang Gung Memorial Hospital, No. 5, Fu-Sing St., Guishan Township, Taoyuan City 333, Taiwan, R.O.C. Tel: +886 33281200 ext.8101, e-mail: victor.wr.lin@gmail.com; Chau-Ting Yeh, MD, Ph.D., Liver Research Center, Linkou Medical Center, Chang Gung Memorial Hospital, No. 5, Fu-Sing St., Guishan Township, Taoyuan City 333, Taiwan, R.O.C. Tel: +88633281200 ext.8101, e-mail: chautingy@gmail.com

Key Words: Direct-acting antiviral (DAA), hepatocellular carcinoma (HCC), hepatitis $\mathrm{C}$ virus (HCV), transarterial chemoembolization (TACE).
Primary liver cancer ranks sixth in cancer incidence $(841,000$ new cases) and fourth in cancer-related mortality $(781,000$ cases) globally. Hepatocellular carcinoma (HCC) is the most common primary liver cancer and comprises over $80 \%$ of all liver tumors. Several known risk factors are linked to development of HCC including chronic viral infection with either hepatitis B virus (HBV) or hepatitis C virus (HCV), heavy alcohol intake, type 2 diabetes, obesity/fatty liver disease, aflatoxin contaminated foodstuffs, hereditary hemochromatosis, alpha1-antitrypsin deficiency, autoimmune hepatitis, some porphyrias, and Wilson's disease (2). These diseases also lead to hepatic fibrosis and cirrhosis. Furthermore, pre-existing cirrhosis is observed in over $80 \%$ of HCC patients.

The Barcelona Clinic Liver Cancer (BCLC) staging classification demonstrates the best predictive efficacy for HCC survival and is used as the universal practice guideline to determine the HCC treatment strategy. For BCLC stage B patients, transcatheter arterial chemoembolization (TACE) is one of the common treatments.

Chronic hepatitis $\mathrm{C}$ (CHC) infection is the second most common cause of HCC globally, which accounts for 10 25\% of cases in Taiwan, and is the most common cause of HCC in Western countries. There are over 184 million people infected with HCV worldwide. CHC is associated with 20 30-fold increased risk for HCC. For HCV related cirrhosis, the annual incidence of $\mathrm{HCC}$ rate is around $3-5 \%$ and the 5-year cumulative risk ranges from $5 \sim 30 \%$. Studies have shown that liver related morbidity and mortality can be reduced after CHC patients have achieved sustained viral response (SVR). As medicine advances, HCV treatment has evolved from pegylated interferon (Peg-IFN) to direct-acting antiviral (DAA) combination regimens which can achieve over $95 \%$ SVR with a low number of adverse events. Current guidelines recommend antiviral treatment for all $\mathrm{CHC}$ patients who do 
not have comorbidity-related decreased life expectancy. The goal of antiviral treatments is to eradicate $\mathrm{CHC}$ infection and reduce its complications including cirrhosis, hepatic decompensation, HCC and mortality.

It is controversial whether DAA treatment for $\mathrm{CHC}$ decreases recurrence of HCC or improves HCC outcome. Reig et al. reported that 19 out of $58 \mathrm{HCV}$ DAA-treated patients who had complete tumor response after anti-HCC treatment had a high tumor recurrence rate of $27.6 \%$, within a median follow-up of 5.7 months. Similar findings were observed by Conti et al. and Ravi et al. who reported $28.81 \%$ and $9.1 \%$ HCC recurrence within 6 months after DAA treatment, respectively. Later studies reported otherwise. Singal et al. observed that DAA-treated and untreated patients had HCC recurrence rate of $42.1 \%$ and $58.9 \%$, respectively; Cabibbo et al. showed no significant difference of HCC recurrence in DAA treated and untreated groups $(\mathrm{HR}=0.70 ; 95 \% \mathrm{CI}=0.44-1.13 ; p=0.15)$.

Furthermore, the role of DAA therapy has not been clearly defined in patients with active intermediate/advanced stage HCC undergoing anti-HCC treatment. The 2020 American Gastroenterological Association (AGA) expert review on DAA and HCC reported that there was insufficient data regarding DAA therapy in patients with active intermediate or advanced stage HCC. Most of current studies were conducted on patients with earlier stage HCC (BCLC 0/A) who can receive curative treatment such as resection, local radiofrequency ablation, and transplantation. The benefit of DAA in CHC patients with intermediate/advanced stage of HCC is not clear. In this retrospective study, we examined the hypothesis that DAA treatment could improve outcomes in CHC patients with HCC BCLC stage B.

\section{Patients and Methods}

Study design and selection of patients. A retrospective cohort study of $\mathrm{CHC}$ patients $\mathrm{CHC}$ with HCC BCLC Stage B receiving TACE at the Linkou medical center of Chang Gung Memorial Hospital between 2010 to 2019 was conducted. This study was conducted in accordance with the ethical standards of our Institution's review board and the 1964 Helsinki declaration and its later amendments or comparable ethical standards. Medical records were reviewed to identify appropriate patients to be included in the study. Baseline demographics and characteristics, including tumor status, tumor number, largest tumor size, tertiary portal vein invasion, and previous surgery/ablation were recorded.

All patients had confirmed $\mathrm{CHC}$ with positive hepatitis $\mathrm{C}$ virus (HCV) RNA test. Diagnosis of HCC was made either pathologically or clinically. Clinical diagnosis was based on characteristic radiologic findings of arterial hypervascularity and venous or delayed phase washout under dynamic multiple detector computed tomography (MDCT) or contrast-magnetic resonance imaging (MRI). For those unable to be diagnosed clinically, liver biopsy was performed for pathological diagnosis (18). HCC staging was assessed according to the modified BCLC classification in 2019
American Association for the Study of Liver Diseases (AASLD) HCC practice guideline. Among 113 patients with $\mathrm{CHC} / \mathrm{HCC}, 14$ patients had received DAA for HCV eradication.

TACE treatment. TACE was performed as the current standard treatment for patients with intermediate stage HCC. Hepatic arterial angiography was performed to identify the target artery of HCC. Doxorubicin 20-60 mg (depending on the tumor size) mixed with 5-20 ml of lipiodol emulsion was infused through the target artery. Further, Gelfoam injection for target artery embolization was performed until no tumor stain was seen on angiography.

TACE treatment response and follow-up. Multiphase CT/MRI was performed 6-10 weeks after each TACE treatment. Treatment response was evaluated using the modified Response Evaluation Criteria in Solid Tumors (mRECIST). Patients were followed until the time of death or loss to follow-up. For patient with complete response after TACE, serum alpha-fetoprotein (AFP) and abdominal ultrasound were monitored every 3 months. In the presence of newly suspected lesions or elevated AFP, further CT/MRI were performed for diagnosis of HCC recurrence.

DAA treatment. Depending on the DAA availability in Taiwan, different regimens were used. Achieving SVR was defined as undetectable $\mathrm{HCV}$ RNA at 12 weeks after completing DAA treatment.

Statistical analysis. Statistical analyses were performed using IBM SPSS statistic software, version 23 (IBM Corporation, Armonk, NY, USA). For the descriptive statistic, categorical data were expressed in number and percentage, whereas the continuous data were presented in mean with standard deviation or median with range. Median overall survival (OS) was calculated in months. KaplanMeier curve was used to evaluate the survival curves. Relevant baseline variables were analyzed using Cox regression hazard model to identify the factors associating with OS, time-to-complete response (TTCR), and progression-free survival (PFS). Statistically significant variables $(\mathrm{p}<0.05)$ in univariate analysis were further included in the multivariate analysis.

\section{Results}

Baseline characteristics of patients. A total of $113 \mathrm{CHC}$ patients who were also diagnosed as HCC BCLC stage B, were included in the study. The basic characteristics of patients are listed in Table I. The mean age was $67.2 \pm 9.3$ years, and 76 of the patients were males. The group was predominantly cirrhotic Child-Pugh A (103/113, 91.2\%). Sixty patients had tumor number $\geq 3$, largest tumor size mean of $3.2 \pm 1.7 \mathrm{~cm}$. All patients had received ongoing TACEs for $\mathrm{HCC}$ as the standard of care according the BCLC guideline, 14 patients also received DAA treatment for $\mathrm{HCV}$ eradication.

Subgroup characteristics. We subdivided the 113 patients into two groups, DAA treatment group and non-treatment group (Table I). When the demographic findings of the two groups were compared, the DAA treatment group had statistically significant better Eastern Cooperative Oncology Group (ECOG) 
Table I. Basic clinical data of 113 HCV/HCC patients receiving TACE treatment.

\begin{tabular}{|c|c|c|c|c|}
\hline Variables & All & HCV tx, n=14 & HCV untx, n=99 & $p$-Value \\
\hline Gender, male, $\%$ & $76(67.3 \%)$ & $9(64.3 \%)$ & $67(67.7 \%)$ & 0.802 \\
\hline Age, years, mean \pm SD & $67.2 \pm 9.3$ & $69.9 \pm 7.1$ & $66.8 \pm 9.5$ & 0.237 \\
\hline$<60$ & & $1(7.1 \%)$ & $27(27.3 \%)$ & \\
\hline$\geq 60$ & & $13(92.9 \%)$ & $72(72.7 \%)$ & \\
\hline ECOG, $\mathrm{PS} \geq 1, \%$ & $30(26.5 \%)$ & $0(0 \%)$ & $27(27.3 \%)$ & 0.043 \\
\hline Cirrhosis, yes, $\%$ & $103(91.2 \%)$ & $10(71.4 \%)$ & $92(92.9 \%)$ & 0.078 \\
\hline Alcoholism, yes, $\%$ & $22(19.5 \%)$ & $0(0 \%)$ & $22(22.2 \%)$ & 0.05 \\
\hline Previous OP/ablation, yes, $\%$ & $38(33.6 \%)$ & $3(21.4 \%)$ & $35(35.4 \%)$ & 0.306 \\
\hline Tertiary PV invasion, yes, $\%$ & $7(6.2 \%)$ & $0(0 \%)$ & $7(0.07 \%)$ & 0.309 \\
\hline Ascites, yes, \% & $19(16.8 \%)$ & $1(0.07 \%)$ & $18(18.1 \%)$ & 0.306 \\
\hline DAA therapy, yes, $\%$ & $14(12.4 \%)$ & $14(100 \%)$ & $0(0 \%)$ & $<0.001$ \\
\hline Tumor number, $\geq 3, \%$ & $60(53.1 \%)$ & $11(78.6 \%)$ & $49(49.5 \%)$ & 0.042 \\
\hline Largest size of tumor, $\mathrm{cm}$, mean $\pm \mathrm{SD}$ & $3.2 \pm 1.7$ & $3.8 \pm 3.0$ & $3.1 \pm 1.4$ & 0.226 \\
\hline $\mathrm{AFP}, \mathrm{ng} / \mathrm{ml}$, median, range & $28.0(2.1-12900.0)$ & $18(3.0-1030.8)$ & $28.0(2.1-12897.9)$ & 0.445 \\
\hline$<100$ & & $11(78.6 \%)$ & $70(70.7 \%)$ & \\
\hline$\geq 100$ & & $3(21.4 \%)$ & $29(29.3 \%)$ & \\
\hline Albumin, $\mathrm{g} / \mathrm{dl}$, mean $\pm \mathrm{SD}$ & $3.5 \pm 0.5$ & $3.9 \pm 0.4$ & $3.4 \pm 0.5$ & 0.001 \\
\hline$<3.5$ & & $3(21.4 \%)$ & $57(57.6 \%)$ & \\
\hline$\geq 3.5$ & & $11(78.6 \%)$ & $42(42.4 \%)$ & \\
\hline Bilirubin, $\mathrm{mg} / \mathrm{dl}$, mean $\pm \mathrm{SD}$ & $1.2 \pm 0.7$ & $1.1 \pm 0.8$ & $1.2 \pm 0.7$ & 0.408 \\
\hline$\leq 1.5$ & & $12(85.7 \%)$ & $82(82.8 \%)$ & \\
\hline$>1.5$ & & $2(14.3 \%)$ & $17(17.2 \%)$ & \\
\hline Prothrombin time, sec, mean \pm SD & $12.4 \pm 1.6$ & $13.0 \pm 2.3$ & $12.3 \pm 1.5$ & 0.157 \\
\hline$\leq 12$ & & $4(28.6 \%)$ & $50(50.5 \%)$ & \\
\hline$>12$ & & $10(71.4 \%)$ & $49(49.5 \%)$ & \\
\hline Creatinine, $\mathrm{mg} / \mathrm{dl}$, mean $\pm \mathrm{SD}$ & $1.0 \pm 0.4$ & $0.8 \pm 0.3$ & $1.0 \pm 0.4$ & 0.142 \\
\hline$\leq 1.0$ & & $11(78.6 \%)$ & $70(70.7 \%)$ & \\
\hline$>1.0$ & & $3(21.4 \%)$ & $29(29.3 \%)$ & \\
\hline $\mathrm{AST}, \mathrm{U} / 1$, mean $\pm \mathrm{SD}$ & $75.0 \pm 57.3$ & $43.6 \pm 24.6$ & $79.5 \pm 59.2$ & 0.027 \\
\hline$\leq 40$ & & $6(42.9 \%)$ & $22(22.2 \%)$ & \\
\hline$>40$ & & $8(57.1 \%)$ & $77(77.8 \%)$ & \\
\hline $\mathrm{ALT}, \mathrm{U} / 1$, mean $\pm \mathrm{SD}$ & $61.3 \pm 47.4$ & $33.9 \pm 25.7$ & $65.2 \pm 48.5$ & 0.02 \\
\hline$\leq 40$ & & $10(71.4 \%)$ & $32(32.3 \%)$ & \\
\hline$>40$ & & $4(28.6 \%)$ & $67(67.7 \%)$ & \\
\hline WBC, $10^{9} / 1$, mean \pm SD & $5.1 \pm 2.1$ & $5.6 \pm 2.0$ & $5.0 \pm 2.1$ & 0.283 \\
\hline $\mathrm{NLR}$, mean $\pm \mathrm{SD}$ & $2.9 \pm 4.6$ & $2.5 \pm 1.7$ & $2.9 \pm 4.9$ & 0.758 \\
\hline Hemoglobin, g/dl, mean \pm SD & $12.3 \pm 1.8$ & $12.8 \pm 1.7$ & $12.2 \pm 1.8$ & 0.302 \\
\hline Platelet, $10^{9} / 1$, mean \pm SD & $122.6 \pm 57.5$ & $138.9 \pm 92.5$ & $120.3 \pm 50.9$ & 0.26 \\
\hline$<100$ & & $5(35.7 \%)$ & $36(36.4 \%)$ & \\
\hline$\geq 100$ & & $9(64.3 \%)$ & $63(63.6 \%)$ & \\
\hline Survival, median, range & $25.1(1.4-158.3)$ & $40.2(12.7-117.7)$ & $22.9(1.4-158.3)$ & \\
\hline Progression, median & $16.5+/ 121.3$ & $13.5+/-12.3$ & & \\
\hline
\end{tabular}

Tx: Treated; untx: untreated; SD: standard deviation; ECOG: Eastern Cooperative Oncology Group; PS: performance status; OP: operation; PV: portal vein; DAA: direct acting antiviral; AFP: alpha-fetoprotein; AST: aspartate transaminase; ALT: alanine transaminase; WBC: white blood cell; NLR: neutrophil/lymphocyte ratio. Significant $p$-Values are shown in bold.

performance status, albumin, aspartate aminotransferase (AST), alanine aminotransferase (ALT). Notably, none of the DAAtreated patients had a history of alcoholism. However, they had significantly more numbers of HCC.

CHC DAA treatments and the overall survival. Out of 113 patients, 14 patients had received various DAA treatments with durations ranging from 8 24 weeks. Two started DAA prior to TACE treatments, and the rest were treated by DAA in parallel with TACE treatment. Thirteen achieved SVR12 (13/14, 92.9\%). Only patient number two had HCV reactivation. The clinical and virological data are listed in Table II. The overall survival in the DAA-treated patients was significantly longer than that in untreated patients as shown in the Kaplan-Meier curve ( $p=0.005$; Figure 1). The median survival was 22.9 months in the untreated $\mathrm{CHC} / \mathrm{HCC}$ patients and 40.2 months in the DAA-treated patients, respectively (Table I). 
Table II. Clinical and virological data of 14 DAA-treated HCV/HCC patients who harbored active HCC tissues and received TACE.

\begin{tabular}{|c|c|c|c|c|c|c|c|c|c|c|c|c|}
\hline Case & Age & Gender & $\mathrm{Cir}^{\mathrm{a}}$ & Child-Pugh & MELD & $\begin{array}{l}\text { HCV RNA } \\
\left(\cdot 10^{6} \mathrm{IU} / \mathrm{ml}\right)\end{array}$ & $\begin{array}{c}\text { HCV } \\
\text { geno-type }\end{array}$ & $\begin{array}{c}\text { DAA } \\
\text { regimen }\end{array}$ & $\begin{array}{c}\text { Treatment } \\
\text { duration } \\
\text { (weeks) }\end{array}$ & $\begin{array}{c}\text { Tumor } \\
\text { number } \\
\text { size }(\mathrm{cm})\end{array}$ & $\begin{array}{c}\text { Largest } \\
\text { tumor } \\
(\mathrm{ng} / \mathrm{ml})\end{array}$ & AFP \\
\hline 1 & 70 & F & $\mathrm{Y}$ & A6 & 8 & 1.381 & $2 \mathrm{a}$ & $\mathrm{SOF}+\mathrm{RBV}$ & 12 & 3 & 2.72 & 29.4 \\
\hline 2 & 63 & F & $\mathrm{Y}$ & B7 & 8 & 5.201 & $1 \mathrm{~b}$ & $3 \mathrm{D}+\mathrm{RBV}$ & 8 & 3 & 1.8 & 1033.8 \\
\hline 3 & 65 & M & $\mathrm{Y}$ & A5 & 7 & 7.499 & $2 b$ & SOF/VEL & 12 & 4 & 1.4 & $<2.0$ \\
\hline 4 & 75 & M & $\mathrm{Y}$ & B9 & 16 & 5.029 & $1 \mathrm{~b}$ & SOF/LED+RBV & 12 & 4 & 1.5 & $<2.0$ \\
\hline 5 & 78 & $\mathrm{~F}$ & $\mathrm{Y}$ & A6 & 8 & 0.361 & $1 \mathrm{~b}$ & SOF/LED & 12 & 4 & 1.4 & 34.3 \\
\hline 6 & 61 & M & Y & A5 & 7 & 5.113 & 6 & $\mathrm{SOF}+\mathrm{RBV}$ & 12 & 3 & 5.8 & 5.3 \\
\hline 7 & 76 & M & $\mathrm{N}$ & A5 & 6 & 7.635 & $1 \mathrm{~b}$ & SOF/LED & 12 & $>4$ & 7.1 & 3.8 \\
\hline 8 & 78 & $\mathrm{~F}$ & $\mathrm{Y}$ & A5 & 7 & 1.446 & $1 \mathrm{~b}$ & SOF/LED & 12 & $>4$ & 4.7 & 188.0 \\
\hline 9 & 75 & M & $\mathrm{Y}$ & A5 & 9 & 0.721 & $1 \mathrm{~b}$ & DAC/ASU & 24 & 3 & 2.4 & 76.4 \\
\hline 10 & 80 & M & $\mathrm{N}$ & A5 & 8 & 0.028 & $1 \mathrm{~b}$ & SOF/LED & 12 & $>4$ & 12.3 & 6.5 \\
\hline 11 & 65 & M & Y & A6 & 7 & 1.554 & $1 \mathrm{~b}$ & $3 \mathrm{D}$ & 12 & 2 & 4.2 & 779.1 \\
\hline 12 & 69 & $\mathrm{~F}$ & $\mathrm{Y}$ & A5 & 10 & 0.040 & $1 \mathrm{~b}$ & $\mathrm{SOF} / \mathrm{DAC}$ & 24 & 3 & 1.7 & 53.8 \\
\hline 13 & 58 & M & $\mathrm{N}$ & A5 & 8 & 0.038 & $1 \mathrm{~b}$ & $3 \mathrm{D}$ & 12 & 2 & 3.5 & 5.1 \\
\hline 14 & 66 & M & Y & A5 & 10 & 7.567 & $2 a+b$ & $\mathrm{SOF}+\mathrm{RBV}$ & 12 & 2 & 3.3 & 3.0 \\
\hline
\end{tabular}

aCir: Clinical cirrhosis, defined as presence of esophageal varices or FibroScan stiffness value >18 kPa; Y: yes; N: no. bSOF: Sofosbuvir; RBV: ribavirin; 3D: dasabuvir/ombitasvir/partaprvir/ritonavir; VEL: velpatasvir; LED: ledipasvir; DAC: daclatasvir; ASU: asunaprevir.

Prognostic factors associated with survival outcomes. Univariate and multivariate analyses by cox proportional hazard model were used to assess various prognostic marks of $\mathrm{CHC} / \mathrm{HCC}$. Among the $113 \mathrm{CHC}$ patients with intermediate stage HCC, several factors, including ECOG performance status, tertiary portal vein invasion, DAA therapy, and serum albumin, were identified to be associated with OS by the univariate analysis (Table III). In multivariate analysis, patients with poor ECOG, graded as ECOC $\geq 1$, had increased hazard on survival $(\mathrm{HR}=1.982,95 \% \mathrm{CI}=1.082$ $3.630, p=0.027)$. Significantly lower risk of death was observed in patients who received DAA treatment $(\mathrm{HR}=0.133,95 \% \mathrm{CI}=0.018-0.976, p=0.047)$ and those with serum albumin level higher than $3.5 \mathrm{ng} / \mathrm{ml}(\mathrm{HR}=0.457$, $95 \% \mathrm{CI}=0.229-0.912, p=0.026)$.

Our univariate analysis revealed that male sex, bilirubin, AST, ALT, white blood counts (WBC), and DAA therapy significantly impacted TTCR (Table IV). Further multivariate analysis showed that bilirubin $(\mathrm{HR}=0.436,95 \% \mathrm{CI}=0.203$ 0.933, $p=0.032)$, ALT $(\mathrm{HR}=0.983,95 \% \mathrm{CI}=0.966-1.000$, $p=0.048)$, and WBC (HR=0.746, 95\% $\mathrm{CI}=0.581-0.958$, $p=0.022$ ) were independent adverse factors influencing TTCR. In addition, DAA treatment was a good prognostic factor for TTCR (HR=3.137, 95\% CI=1.334-7.378, $p=0.009)$.

For the PFS (Table V), univariate analysis identified previous HCC treatments, tumor size, AFP, WBC, and platelet counts (PLT) as significant factors. However, multivariate analyses further showed previous HCC treatments $\quad(\mathrm{HR}=2.291, \quad 95 \% \mathrm{CI}=1.394-3.766), \quad \mathrm{AFP}$ $(\mathrm{HR}=1.021,95 \% \mathrm{CI}=1.004-1.039)$, and $\mathrm{WBC}(\mathrm{HR}=1.180$,

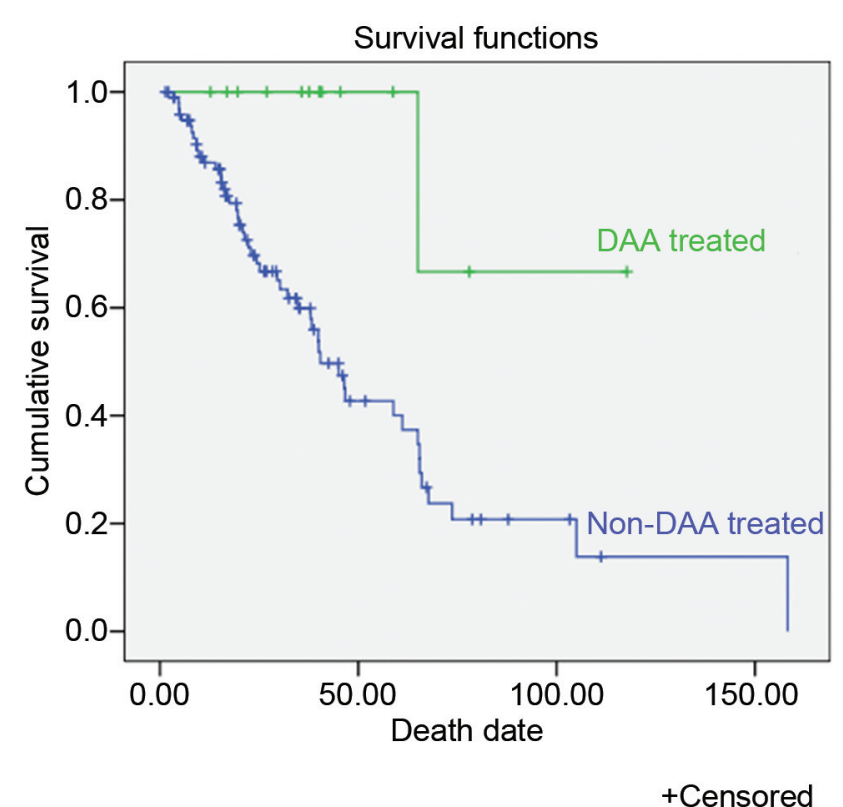

Figure 1. Kaplan-Meier curve of overall survival between direct-acting antiviral (DAA)-treated and non-DAA treated patients.

95\% CI=1.056-1.319) were associated with increased risk for tumor progression.

\section{Discussion}

Current guidelines only advice DAA therapy in $\mathrm{CHC} / \mathrm{HCC}$ BCLC stage 0/A patients who had received curative HCC 
Table III. Cox proportional hazard analysis for overall survival.

\begin{tabular}{|c|c|c|c|c|}
\hline \multirow[b]{2}{*}{ Variables } & \multicolumn{2}{|c|}{ Univariate } & \multicolumn{2}{|c|}{ Multivariate } \\
\hline & HR $(95 \% \mathrm{CI})$ & $p$-Value & HR $(95 \% \mathrm{CI})$ & $p$-Value \\
\hline Gender, Male $=1$ & $0.882(0.486,1.599)$ & 0.679 & & \\
\hline Age, per year & $1.017(0.984,1.051)$ & 0.317 & & \\
\hline ECOG, performance score $\geq 1$ & $1.987(1.103,3.580)$ & 0.022 & $1.982(1.082,3.630)$ & 0.027 \\
\hline Cirrhosis, Yes=1 & $5.220(0.719,37.896)$ & 0.102 & & \\
\hline Alcoholism, Yes=1 & $1.086(0.540,2.183)$ & 0.817 & & \\
\hline Previous operation/Ablation, Yes=1 & $1.670(0.947,2.945)$ & 0.077 & & \\
\hline Tertiary PV invasion, Yes $=1$ & $3.065(1.079,8.707)$ & 0.036 & $2.405(0.807,7.170)$ & 0.115 \\
\hline Ascites, Yes $=1$ & $1.237(0.575,2.662)$ & 0.586 & & \\
\hline Anti-HCV DAA treatment, Yes $=1$ & $0.100(0.014,0.725)$ & 0.023 & $0.133(0.018,0.976)$ & 0.047 \\
\hline Tumor number, $\geq 3$ as 1 & $0.882(0.503,1.548)$ & 0.662 & & \\
\hline Largest tumor size, per $\mathrm{cm}$ & $0.990(0.8009,1.211)$ & 0.919 & & \\
\hline AFP, per $100 \mathrm{ng} / \mathrm{ml}$ & $0.992(0.973,1.012)$ & 0.426 & & \\
\hline Albumin, per g/dl & $0.321(0.167,0.617)$ & 0.001 & $0.457(0.229,0.912)$ & 0.026 \\
\hline Bilirubin, per mg/dl & $1.166(0.793,1.715)$ & 0.434 & & \\
\hline Prothrombin time, per sec & $0.934(0.763,1.142)$ & 0.503 & & \\
\hline Creatinine, per $\mathrm{mg} / \mathrm{dl}$ & $0.811(0.409,1.612)$ & 0.551 & & \\
\hline AST, per U/1 & $1.003(0.999,1.007)$ & 0.192 & & \\
\hline ALT, per U/1 & $0.998(0.992,1.005)$ & 0.614 & & \\
\hline WBC, per $10^{9} / 1$ & $1.069(0.924,1.238)$ & 0.368 & & \\
\hline Neutrophil/Lymphocyte ratio, per fold & $1.031(0.984,1.079)$ & 0.197 & & \\
\hline Hemoglobin, per g/dl & $0.958(0.815,1.127)$ & 0.606 & & \\
\hline Platelet, per $10^{9} / 1$ & $1.000(0.995,1.005)$ & 0.925 & & \\
\hline
\end{tabular}

ECOG: Eastern Cooperative Oncology Group; PV: portal vein; HCV: hepatitis C virus; DAA: direct acting antiviral; AFP: alpha-fetoprotein; AST: aspartate transaminase; ALT: alanine transaminase; WBC: white blood cell. Significant $p$-Values are shown in bold.

treatments. It is unclear whether DAA therapy impacts prognosis of patients with intermediate stage HCC. Our retrospective study showed that ECOG, DAA therapy, and serum albumin were independent prognostic factors for intermediate stage $\mathrm{HCC} / \mathrm{CHC}$ patients. We also found that DAA therapy has a positive effect on TTCR. In contrary, male sex, higher bilirubin, ALT, and WBC were associated with poor TACE treatment response. In patients who had previous HCC treatments, higher AFP and WBC were indicators of poor progression-free survival.

Similar to data recorded in various types of malignancy and diseases, it is not surprising that performance status (PS) and hypoalbuminemia are significant indicators of survival outcomes. Patients who have worse PS tend to be frail and are less likely to tolerate rigorous anti-cancer therapy. They are prone to have less favorable outcomes than patients with better PS. Thus, this is probably also the reason that PS is one of the three assessing components for the BCLC HCC staging. Serum albumin is not only an indicator of nutrition status, it is also a liver function parameter that is included in several scoring criteria such as the Child-Pugh Scoring system and Albumin-Bilirubin (ALBI). Our univariate and multivariate analyses showed comparable results.

In the newly established $10^{\text {th }}$ Annual Asia-Pacific Primary liver cancer Expert (APPLE) consensus, it has been stated that liver function preservation is as important as achieving high objective response for the treatment of intermediatestage of HCC. CHC induced liver inflammation causes deterioration of liver functions and results in limited HCC treatment options. Studies have shown that eradication of $\mathrm{HCV}$ can improve liver function, promote fibrosis regression, prevent cirrhosis progression, and reduce risk of liver decompensation, which further result in decreased liverrelated mortality. Current guidelines recommend $\mathrm{HCV}$ eradication for all patients except for those with limited life expectancy. The benefits of $\mathrm{HCV}$ clearance with DAA therapy after curative treatments have been extensively studied in HCC BLCL stage 0 and A patients. However, data regarding whether anti-HCV treatment should be initiated on intermediate or later stage $\mathrm{HCC}$ is currently unknown. Our rationale is that if $\mathrm{HCV}$ clearance can be achieved with DAA therapy, liver dysfunction may be reversed, which might further improve survival even in HCC BCLC stage B patients. Our study showed that DAA therapy had a beneficial effect on overall outcome. It is known that TACE is the standard treatment for intermediate stage HCC, whereas systemic therapy is the treatment of choice for those refractory to TACE. Furthermore, a recent randomized multicenter prospective study conducted by Kudo et al. 
Table IV. Cox proportional hazard analysis for time-to-complete response.

\begin{tabular}{|c|c|c|c|c|}
\hline \multirow[b]{2}{*}{ Variables } & \multicolumn{2}{|c|}{ Univariate } & \multicolumn{2}{|c|}{ Multivariate } \\
\hline & HR (95\%CI) & $p$-Value & HR $(95 \% \mathrm{CI})$ & $p$-Value \\
\hline Gender, Male $=1$ & $0.434(0.214,0.880)$ & 0.021 & $0.678(0.313,1.468)$ & 0.324 \\
\hline Age, per year & $1.003(0.966,1.042)$ & 0.874 & & \\
\hline ECOG, performance score $\geq 1$ & $1.159(0.519,2.589)$ & 0.718 & & \\
\hline Cirrhosis, Yes=1 & $1.070(0.325,3.517)$ & 0.911 & & \\
\hline Alcoholism, Yes=1 & $0.733(0.282,1.904)$ & 0.523 & & \\
\hline Previous operation/Ablation, Yes $=1$ & $0.972(0.460,2.056)$ & 0.941 & & \\
\hline Tertiary PV invasion, Yes=1 & $0.045(0.000,21.752)$ & 0.325 & & \\
\hline Ascites, Yes $=1$ & $0.956(0.368,2.485)$ & 0.927 & & \\
\hline Anti-HCV DAA treatment, Yes $=1$ & $3.828(1.806,8.111)$ & $<0.001$ & $3.137(1.334,7.378)$ & 0.009 \\
\hline Tumor number, $\geq 3$ & $0.825(0.412,1.652)$ & 0.587 & & \\
\hline Largest tumor size, per $\mathrm{cm}$ & $0.917(0.719,1.170)$ & 0.488 & & \\
\hline AFP, per $100 \mathrm{ng} / \mathrm{ml}$ & $1.008(0.985,1.031)$ & 0.521 & & \\
\hline Albumin, per g/dl & $1.445(0.710,2.939)$ & 0.310 & & \\
\hline Bilirubin, per mg/dl & $0.434(0.195,0.965)$ & 0.041 & $0.436(0.203,0.933)$ & 0.032 \\
\hline Prothrombin time, per sec & $0.982(0.797,1.210)$ & 0.867 & & \\
\hline Creatinine, per mg/dl & $0.545(0.163,1.822)$ & 0.324 & & \\
\hline AST, per U/l & $0.989(0.979,0.999)$ & 0.029 & $1.005(0.993,1.018)$ & 0.425 \\
\hline ALT, per U/1 & $0.982(0.969,0.994)$ & 0.005 & $0.983(0.966,1.000)$ & 0.048 \\
\hline WBC, per $10^{9} / 1$ & $0.787(0.640,0.968)$ & 0.023 & $0.746(0.581,0.958)$ & 0.022 \\
\hline Neutrophil/Lymphocyte ratio, per fold & $0.915(0.781,1.073)$ & 0.276 & & \\
\hline Hemoglobin, per g/dl & $0.887(0.742,1.059)$ & 0.185 & & \\
\hline Platelet, per $10^{9} / 1$ & $0.994(0.987,1.001)$ & 0.080 & & \\
\hline
\end{tabular}

ECOG: Eastern Cooperative Oncology Group; PV: portal vein; HCV: hepatitis C virus; DAA: direct acting antiviral; AFP: alpha-fetoprotein; AST: aspartate transaminase; ALT: alanine transaminase; WBC: white blood cell. Significant $p$-Values are shown in bold.

showed that combination treatment with TACE plus sorafenib in unresectable HCC significantly improved progression free survival compared to TACE alone. However, poor liver function and its related complications may hinder eligibility to TACE treatments or systemic therapy. It is important to preserve liver function in these patients to maintain their eligibility for not only subsequent systemic therapy after TACE failure/refractory HCC but also for new combination therapy with TACE and systemic therapy. In this respect, it is strategic and critical to treat HCV with DAA in patients with BCLC stage B HCC.

In patients receiving TACE, tumor response based on mRECIST especially initial complete response (CR) can predict a longer survival benefit. Our study revealed that DAA therapy has a positive effect on TTCR. This may be the reason DAA could improve OS and further support the use of DAA in HCV patients with BCLC stage B HCC.

$\mathrm{Ng}$ et al. found that female gender tends to have HCC survival advantage over male which is likely due to the differences in tumor nature between genders. On the other hand, underlying differences in gene expression in opposite sex may also play a role. Liu et al. identified that Family with sequence similarity 83 (FAM83D) over-expression was observed significantly more in male and was associated with worse outcomes. These findings may provide possible explanations why the TACE treatment response was worse in male patients in the present study.

Studies have shown that poor liver function reserve, highdose chemotherapeutic agents, and history of multiple embolization procedures increased risk of acute liver failure after TACE. Jeon et al. also demonstrated higher total bilirubin levels increased risk of acute hepatic failure after TACE. In our study, higher bilirubin and ALT levels were associated with decreased response to TACE. Both higher levels of bilirubin and ALT were indicators of poor liver dysfunction. Patients with higher bilirubin and ALT levels may be less tolerant to higher dosage of TACE due to higher risk of post-TACE irreversible liver parenchymal injury or even liver failure, thus affecting treatment outcome and leading to a less favorable response.

Tumor progression from previously treated residual HCC and/or repeated tumor recurrence after TACE may be associated with increased malignant potential of the tumor nature. Sustained liver parenchymal injury from previous locoregional treatments may further adversely affect progression-free survival as was seen in our analysis.

Several reports have identified AFP as an independent factor for HCC recurrence. Chen et al. have identified the 
Table V. Cox proportional hazard analysis for progression free survival.

\begin{tabular}{|c|c|c|c|c|}
\hline \multirow[b]{2}{*}{ Variables } & \multicolumn{2}{|c|}{ Univariate } & \multicolumn{2}{|c|}{ Multivariate } \\
\hline & HR $(95 \% \mathrm{CI})$ & $p$-Value & HR $(95 \% \mathrm{CI})$ & $p$-Value \\
\hline Gender, Male $=1$ & $1.266(0.776,2.065)$ & 0.345 & & \\
\hline Age, per year & $0.992(0.967,1.017)$ & 0.515 & & \\
\hline ECOG, performance status $\geq 1$ & $1.057(0.623,1.792)$ & 0.837 & & \\
\hline Cirrhosis, Yes=1 & $1.232(0.565,2.684)$ & 0.600 & & \\
\hline Alcoholism, Yes=1 & $1.168(0.663,2.057)$ & 0.591 & & \\
\hline Previous operation/Ablation, Yes $=1$ & $1.713(1.077,2.781)$ & 0.023 & $2.291(1.394,3.766)$ & 0.001 \\
\hline Tertiary PV invasion, Yes=1 & $1.666(0.669,4.153)$ & 0.273 & & \\
\hline Ascites, Yes $=1$ & $1.138(0.624,2.078)$ & 0.673 & & \\
\hline Anti-HCV DAA treatment, Yes $=1$ & $1.464(0.786,2.728)$ & 0.230 & & \\
\hline Tumor number, $\geq 3$ as 1 & $1.249(0.798,1.956)$ & 0.330 & & \\
\hline Largest tumor size, per $\mathrm{cm}$ & $1.230(1.078,1.404)$ & 0.002 & $1.137(0.969,1.335)$ & 0.116 \\
\hline AFP, per $100 \mathrm{ng} / \mathrm{ml}$ & $1.021(1.004,1.039)$ & 0.017 & $1.021(1.004,1.039)$ & 0.015 \\
\hline Albumin, per g/dl & $1.178(0.734,1.891)$ & 0.498 & & \\
\hline Bilirubin, per mg/dl & $0.878(0.617,1.251)$ & 0.472 & & \\
\hline Prothrombin time, per sec & $0.974(0.836,1.136)$ & 0.737 & & \\
\hline Creatinine, per mg/dl & $0.867(0.489,1.538)$ & 0.625 & & \\
\hline AST, per $\mathrm{U} / 1$ & $1.000(0.996,1.004)$ & 0.995 & & \\
\hline ALT, per $\mathrm{U} / 1$ & $1.000(0.996,1.004)$ & 0.866 & & \\
\hline WBC, per $10^{9} / 1$ & $1.192(1.084,1.311)$ & $<0.001$ & $1.180(1.056,1.319)$ & 0.004 \\
\hline Neutrophil/Lymphocyte ratio, per fold & $1.027(0.987,1.069)$ & 0.192 & & \\
\hline Hemoglobin, per g/dl & $1.034(0.909,1.178)$ & 0.608 & & \\
\hline Platelet, per $10^{9} / 1$ & $1.004(1.000,1.008)$ & 0.038 & $1.002(0.998,1.007)$ & 0.351 \\
\hline
\end{tabular}

ECOG: Eastern Cooperative Oncology Group; PV: portal vein; HCV: hepatitis C virus; DAA: direct acting antiviral; AFP: alpha-fetoprotein; AST: aspartate transaminase; ALT: alanine transaminase; WBC: white blood cell. Significant $p$-Values are shown in bold.

pro-oncogenic role of AFP in HCC progression (44). It is therefore not surprising to observe that higher AFP levels are an unfavorable prognostic factor of PFS in the present study.

Previously, studies have revealed the association between the pro-tumor and anti-tumor inflammatory effects of immune response with elevated WBC and neutrophil-lymphocyte ration (NLR) in different cancers. In our study, elevated WBC and NLR are both associated with unfavorable outcomes. However, NLR did not reach statistical significance, only WBC was found to be a significant factor for TTCR and PFS.

A previous study reported a lower SVR rate with DAA therapy in active HCC compared to inactive HCC (85.5\% vs. $93.7 \%)$. In the present study, we achieved a much higher SVR of $92.9 \%$ in patients with BCLC stage B HCC. However, this may be due to the fact that we had a very small sample size of 14 patients who received DAA.

Our study had several limitations. First, it was a retrospective evaluation from a single medical center in Taiwan. The study population had little heterogeneity. The fact that the entire study cohort was ethnically Taiwanese could contribute to potential selection bias. This may limit the applicability of the results as it is unclear whether similar results would be observed in different ethnic groups. Second, the sample size of 14 patients who ultimately received DAA therapy was small. This limits the statistical power of the study results. However, this was an anticipated problem as the current guidelines do not support the use of DAA for $\mathrm{HCV}$ eradication in HCC BCLC stage B patients. Lastly, of the 14 patients treated by DAA, 12 were Child-Pugh A $(85.7 \%)$. We could not draw conclusions on the benefits of DAA treatment in HCC BLCL stage B patients who had more advanced liver disease. Currently, Child-Push B/C score is listed as contraindication of some DAA therapies.

In conclusion, we demonstrated that DAA therapy was associated with improved OS and TTCR in CHCintermediate stage HCC. Moreover, we demonstrated that ECOG, higher serum albumin, and DAA therapy are independent predictors of survival in $\mathrm{CHC}-\mathrm{HCC}$ patients.

\section{Conflicts of Interest}

All Authors declare no conflicts of interest regarding this article.

\section{Authors' Contributions}

Conception and design: Chau-Ting Yeh; Administrative support: Wey-Ran Lin, Chau-Ting Yeh; Provision of study materials or patients: Wei-Ting Chen, Cheng-Chun Lin, Wey-Ran Lin, ChauTing Yeh; Collection and assembly of data: Changhan Shao; Data 
analysis and interpretation: Changhan Shao, Paul P Shao; Manuscript writing: All Authors; Final approval of manuscript: All Authors.

\section{Acknowledgements}

The Authors would like to thank the members of the Liver Research center for their diligent data collection.

\section{References}

1 Bray F, Ferlay J, Soerjomataram I, Siegel RL, Torre LA and Jemal A: Global cancer statistics 2018: GLOBOCAN estimates of incidence and mortality worldwide for 36 cancers in 185 countries. CA Cancer J Clin 68(6): 394-424, 2018. PMID: 30207593. DOI: $10.3322 /$ caac. 21492

2 El-Serag HB: Hepatocellular carcinoma. N Engl J Med 365(12): 1118-1127, 2011. PMID: 21992124. DOI: 10.1056/NEJ Mra1001683

3 Jindal A, Thadi A and Shailubhai K: Hepatocellular Carcinoma: Etiology and current and future drugs. journal of clinical and experimental hepatology 9(2): 221-232, 2020. DOI: 10.1016/j.jceh.2019.01.004

4 Llovet JM, Brú C and Bruix J: Prognosis of hepatocellular carcinoma: The BCLC staging classification. Semin Liver Dis 19(3): 329-338, 1999. PMID: 10518312. DOI: $10.1055 / \mathrm{s}-2007-$ 1007122

5 Marrero JA, Kulik LM, Sirlin CB, Zhu AX, Finn RS, Abecassis MM, Roberts LR and Heimbach JK: Diagnosis, staging, and management of hepatocellular carcinoma: 2018 practice guidance by the american association for the study of liver diseases. Hepatology 68(2): 723-750, 2018. PMID: 29624699. DOI: $10.1002 /$ hep. 29913

6 Ghouri YA, Mian I and Rowe JH: Review of hepatocellular carcinoma: Epidemiology, etiology, and carcinogenesis. J Carcinog 16: 1, 2017. PMID: 28694740. DOI: 10.4103/jcar.JCar_9_16

7 Mohd Hanafiah K, Groeger J, Flaxman AD and Wiersma ST: Global epidemiology of hepatitis $C$ virus infection: new estimates of age-specific antibody to HCV seroprevalence. Hepatology 57(4): 1333-1342, 2013. PMID: 23172780. DOI: 10.1002/hep.26141

8 Singal AG, Volk ML, Jensen D, Di Bisceglie AM and Schoenfeld PS: A sustained viral response is associated with reduced liver-related morbidity and mortality in patients with hepatitis C virus. Clin Gastroenterol Hepatol 8(3): 280-8, 288.e1, 2010. PMID: 19948249. DOI: 10.1016/j.cgh.2009.11.018

9 Ghany MG, Morgan TR and AASLD-IDSA Hepatitis C Guidance Panel.: Hepatitis C guidance 2019 update: American association for the study of liver diseases-infectious diseases society of America recommendations for testing, managing, and treating Hepatitis C virus infection. Hepatology 71(2): 686-721, 2020. PMID: 31816111 . DOI: $10.1002 /$ hep. 31060

10 European Association for the Study of the Liver. Electronic address: easloffice@easloffice.eu. and European Association for the Study of the Liver.: EASL Recommendations on Treatment of Hepatitis C 2018. J Hepatol 69(2): 461-511, 2018. PMID: 29650333. DOI: $10.1016 /$ j.jhep.2018.03.026

11 World Health Organization: Global health sector strategy on viral hepatitis 2016-2021. Towards ending viral hepatitis: World Health
Organization, 2016. Available at: https://apps.who.int/iris/bitstream/ handle/10665/246177/WHO-HIV-2016.06-eng.pdf; jsessionid=DE64F4054D37D6316D0D6BE350305394? sequence $=1$ [Last accessed on February 19, 2021]

12 Reig M, Mariño Z, Perelló C, Iñarrairaegui M, Ribeiro A, Lens S, Díaz A, Vilana R, Darnell A, Varela M, Sangro B, Calleja JL, Forns $\mathrm{X}$ and Bruix $\mathrm{J}$ : Unexpected high rate of early tumor recurrence in patients with HCV-related HCC undergoing interferon-free therapy. J Hepatol 65(4): 719-726, 2016. PMID: 27084592. DOI: 10.1016/j.jhep.2016.04.008

13 Conti F, Buonfiglioli F, Scuteri A, Crespi C, Bolondi L, Caraceni P, Foschi FG, Lenzi M, Mazzella G, Verucchi G, Andreone P and Brillanti S: Early occurrence and recurrence of hepatocellular carcinoma in HCV-related cirrhosis treated with direct-acting antivirals. J Hepatol 65(4): 727-733, 2016. PMID: 27349488. DOI: $10.1016 / \mathrm{j}$.jhep.2016.06.015

14 Ravi S, Axley P, Jones D, Kodali S, Simpson H, McGuire BM and Singal AK: Unusually high rates of hepatocellular carcinoma after treatment with direct-acting antiviral therapy for hepatitis c related cirrhosis. Gastroenterology 152(4): 911-912, 2017. PMID: 28161225. DOI: 10.1053/j.gastro.2016.12.021

15 Singal AG, Rich NE, Mehta N, Branch A, Pillai A, Hoteit M, Volk M, Odewole M, Scaglione S, Guy J, Said A, Feld JJ, John BV, Frenette C, Mantry P, Rangnekar AS, Oloruntoba O, Leise M, Jou JH, Bhamidimarri KR, Kulik L, Tran T, Samant H, Dhanasekaran R, Duarte-Rojo A, Salgia R, Eswaran S, Jalal P, Flores A, Satapathy SK, Wong R, Huang A, Misra S, Schwartz M, Mitrani R, Nakka S, Noureddine W, Ho C, Konjeti VR, Dao A, Nelson K, Delarosa K, Rahim U, Mavuram M, Xie JJ, Murphy CC and Parikh ND: Direct-acting antiviral therapy not associated with recurrence of hepatocellular carcinoma in a multicenter north american cohort study. Gastroenterology 156(6): 1683-1692.e1, 2019. PMID: 30660729. DOI: 10.1053/j.gastro.2019.01.027

16 Cabibbo G, Celsa C, Calvaruso V, Petta S, Cacciola I, Cannavò MR, Madonia S, Rossi M, Magro B, Rini F, Distefano M, Larocca L, Prestileo T, Malizia G, Bertino G, Benanti F, Licata A, Scalisi I, Mazzola G, Di Rosolini MA, Alaimo G, Averna A, Cartabellotta F, Alessi N, Guastella S, Russello M, Scifo G, Squadrito G, Raimondo G, Trevisani F, Craxì A, Di Marco V, Cammà C and Rete Sicilia Selezione Terapia - HCV (RESIST-HCV) and Italian Liver Cancer (ITA.LI.CA.) Group.: Direct-acting antivirals after successful treatment of early hepatocellular carcinoma improve survival in HCV-cirrhotic patients. J Hepatol 71(2): 265-273, 2019. PMID: 30959157. DOI: 10.1016/j.jhep.2019.03.027

17 Singal AG, Lim JK and Kanwal F: AGA clinical practice update on interaction between oral direct-acting antivirals for chronic hepatitis $\mathrm{C}$ infection and hepatocellular carcinoma: Expert Review. Clin Liver Dis (Hoboken) 15(6): 211-212, 2020. PMID: 32617150. DOI: $10.1002 /$ cld.943

18 Surveillance group., Diagnosis group., Staging group., Surgery group., Local ablation group., TACE/TARE/HAI group., Target therapy/systemic therapy group., Radiotherapy group., Prevention group. and Drafting group.: Management consensus guideline for hepatocellular carcinoma: 2016 updated by the Taiwan Liver Cancer Association and the Gastroenterological Society of Taiwan. J Formos Med Assoc 117(5): 381-403, 2018. PMID: 29074347. DOI: 10.1016/j.jfma.2017.09.007

19 Lencioni R and Llovet JM: Modified RECIST (mRECIST) assessment for hepatocellular carcinoma. Semin Liver Dis 30(1): 52-60, 2010. PMID: 20175033. DOI: 10.1055/s-0030-1247132 
20 Oken MM, Creech RH, Tormey DC, Horton J, Davis TE, McFadden ET and Carbone PP: Toxicity and response criteria of the Eastern Cooperative Oncology Group. Am J Clin Oncol 5(6): 649-655, 1982. PMID: 7165009

21 Laird BJ, Kaasa S, McMillan DC, Fallon MT, Hjermstad MJ, Fayers P and Klepstad P: Prognostic factors in patients with advanced cancer: A comparison of clinicopathological factors and the development of an inflammation-based prognostic system. Clin Cancer Res 19(19): 5456-5464, 2013. PMID: 23938289. DOI: $10.1158 / 1078-0432$.CCR-13-1066

22 Gupta D and Lis CG: Pretreatment serum albumin as a predictor of cancer survival: A systematic review of the epidemiological literature. Nutr J 9: 69, 2010. PMID: 21176210. DOI: 10.1186/ 1475-2891-9-69

23 Akirov A, Masri-Iraqi H, Atamna A and Shimon I: Low albumin levels are associated with mortality risk in hospitalized patients. Am J Med 130(12): 1465.e11-1465.e19, 2017. PMID: 28803138 DOI: 10.1016/j.amjmed.2017.07.020

24 Tojek K, Kowalczyk G, Czerniak B, Banaś W, Szukay B, Korzycka-Wilińska W, Banaszkiewicz Z and Budzyński J: Blood albumin as a prognostic factor among unselected medically treated inpatients. Biomark Med 13(13): 1059-1069, 2019. PMID: 31475857. DOI: 10.2217/bmm-2018-0465

25 Child CG and Turcotte JG: Surgery and portal hypertension. Major Probl Clin Surg 1: 1-85, 1964. PMID: 4950264

26 Johnson PJ, Berhane S, Kagebayashi C, Satomura S, Teng M, Reeves HL, O'Beirne J, Fox R, Skowronska A, Palmer D, Yeo W, Mo F, Lai P, Iñarrairaegui M, Chan SL, Sangro B, Miksad R, Tada T, Kumada $\mathrm{T}$ and Toyoda H: Assessment of liver function in patients with hepatocellular carcinoma: a new evidence-based approach-the ALBI grade. J Clin Oncol 33(6): 550-558, 2015. PMID: 25512453. DOI: 10.1200/JCO.2014.57.9151

27 Pugh RN, Murray-Lyon IM, Dawson JL, Pietroni MC and Williams R: Transection of the oesophagus for bleeding oesophageal varices. Br J Surg 60(8): 646-649, 1973. PMID: 4541913. DOI: $10.1002 /$ bjs.1800600817

28 Kudo M, Han KH, Ye SL, Zhou J, Huang YH, Lin SM, Wang CK, Ikeda M, Chan SL, Choo SP, Miyayama S, Cheng AL. A changing paradigm for the treatment of intermediate-stage hepatocellular carcinoma: Asia-Pacific Primary Liver Cancer Expert Consensus Statements. Liver Cancer. 2020 Jun;9(3):245260. doi: 10.1159/000507370. Epub 2020 May 13. PMID: 32647629

29 Backus LI, Boothroyd DB, Phillips BR, Belperio P, Halloran J and Mole LA: A sustained virologic response reduces risk of allcause mortality in patients with hepatitis C. Clin Gastroenterol Hepatol 9(6): 509-516.e1, 2011. PMID: 21397729. DOI: 10.1016/j.cgh.2011.03.004

30 Rutter K, Stättermayer AF, Beinhardt S, Scherzer TM, SteindlMunda P, Trauner M, Ferenci P and Hofer H: Successful antiviral treatment improves survival of patients with advanced liver disease due to chronic hepatitis C. Aliment Pharmacol Ther 41(6): 521-531, 2015. PMID: 25616017. DOI: 10.1111/ apt. 13085

31 van der Meer AJ, Veldt BJ, Feld JJ, Wedemeyer H, Dufour J-F, Lammert F, Duarte-Rojo A, Heathcote EJ, Manns MP and Kuske L: Association between sustained virological response and allcause mortality among patients with chronic hepatitis $\mathrm{c}$ and advanced hepatic fibrosis. JAMA 308(24): 2584-2593, 2012. PMID: 23268517. DOI: 10.1001/jama.2012.144878
32 Cabibbo G, Petta S, Barbara M, Attardo S, Bucci L, Farinati F, Giannini EG, Negrini G, Ciccarese F, Rapaccini GL, Di Marco M, Caturelli E, Zoli M, Borzio F, Sacco R, Virdone R, Marra F, Mega A, Morisco F, Benvegnù L, Gasbarrini A, Svegliati-Baroni G, Foschi FG, Olivani A, Masotto A, Nardone G, Colecchia A, Persico M, Craxì A, Trevisani $\mathrm{F}$, Cammà $\mathrm{C}$ and Italian Liver Cancer (ITA.LI.CA) group.: Hepatic decompensation is the major driver of death in HCV-infected cirrhotic patients with successfully treated early hepatocellular carcinoma. J Hepatol 67(1): 65-71, 2017. PMID: 28192185. DOI: 10.1016/j.jhep.2017.01.033

33 Kudo M, Ueshima K, Ikeda M, Torimura T, Tanabe N, Aikata H, Izumi N, Yamasaki T, Nojiri S, Hino K, Tsumura H, Kuzuya T, Isoda N, Yasui K, Aino H, Ido A, Kawabe N, Nakao K, Wada Y, Yokosuka O, Yoshimura K, Okusaka T, Furuse J, Kokudo N, Okita K, Johnson PJ, Arai Y and TACTICS study group.: Randomised, multicentre prospective trial of transarterial chemoembolisation (TACE) plus sorafenib as compared with TACE alone in patients with hepatocellular carcinoma: TACTICS trial. Gut 69(8): 1492-1501, 2020. PMID: 31801872. DOI: $10.1136 /$ gutjnl-2019-318934

34 Kim BK, Kim SU, Kim KA, Chung YE, Kim MJ, Park MS, Park JY, Kim DY, Ahn SH, Kim MD, Park SI, Won JY, Lee DY and $\mathrm{Han} \mathrm{KH}$ : Complete response at first chemoembolization is still the most robust predictor for favorable outcome in hepatocellular carcinoma. J Hepatol 62(6): 1304-1310, 2015. PMID: 25637785. DOI: 10.1016/j.jhep.2015.01.022

35 Zhang Y, Zhang M, Chen M, Mei J, Xu L, Guo R, Lin X, Li J and Peng Z: Association of Sustained Response Duration With Survival After Conventional Transarterial Chemoembolization in Patients With Hepatocellular Carcinoma. JAMA Netw Open 1(6): e183213, 2018. PMID: 30646226. DOI: 10.1001/ jamanetworkopen.2018.3213

$36 \mathrm{Ng}$ IO, Ng MM, Lai EC and Fan ST: Better survival in female patients with hepatocellular carcinoma. Possible causes from a pathologic approach. Cancer 75(1): 18-22, 1995. PMID: 7804971. DOI: 10.1002/1097-0142(19950101)75:1<18::aidcncr2820750105>3.0.co;2-\#

37 Liu X, Gao H, Zhang J and Xue D: FAM83D is associated with gender, AJCC stage, overall survival and disease-free survival in hepatocellular carcinoma. Biosci Rep 39(5): 2019. PMID: 30910840. DOI: 10.1042/BSR20181640

38 Katsushima S, Inokuma T, Oi H, Okamura J, Higashi T, Takeuchi R, Hidaka A, Shigeno C, Iida Y and Konishi J: Acute hepatic failure following transcatheter arterial embolization for the treatment of hepatocellular carcinoma. Digestion 58(2): 189195, 1997. PMID: 9144310. DOI: 10.1159/000201443

39 Jeon SH, Park KS, Kim YH, Shin YS, Kang MK, Jang BK, Chung WJ, Cho KB and Hwang JS: [Incidence and risk factors of acute hepatic failure after transcatheter arterial chemoembolization for hepatocellular carcinoma]. Korean J Gastroenterol 50(3): 176-182, 2007. PMID: 17885283

40 Shim JH, Park JW, Kim JH, An M, Kong SY, Nam BH, Choi JI, Kim HB, Lee WJ and Kim CM: Association between increment of serum VEGF level and prognosis after transcatheter arterial chemoembolization in hepatocellular carcinoma patients. Cancer Sci 99(10): 2037-2044, 2008. PMID: 19016764. DOI: 10.1111/j.1349-7006.2008.00909.x

41 Kong J, Kong J, Pan B, Ke S, Dong S, Li X, Zhou A, Zheng L and Sun WB: Insufficient radiofrequency ablation promotes angiogenesis of residual hepatocellular carcinoma via HIF- 
1 $\alpha$ /VEGFA. PLoS One 7(5): e37266, 2012. PMID: 22615958. DOI: $10.1371 /$ journal.pone.0037266

42 Lleo A, Aglitti A, Aghemo A, Maisonneuve P, Bruno S, Persico $\mathrm{M}$ and collaborators.: Predictors of hepatocellular carcinoma in $\mathrm{HCV}$ cirrhotic patients treated with direct acting antivirals. Dig Liver Dis 51(2): 310-317, 2019. PMID: 30473220. DOI: 10.1016/j.dld.2018.10.014

43 Yoshimasu Y, Furuichi Y, Kasai Y, Takeuchi H, Sugimoto K, Nakamura I and Itoi T: Predictive factors for hepatocellular carcinoma occurrence or recurrence after direct-acting antiviral agents in patients with chronic hepatitis C. J Gastrointestin Liver Dis 28(1): 63-71, 2019. PMID: 30851174. DOI: 10.15403/ jgld.2014.1121.281.hpc

44 Chen T, Dai X, Dai J, Ding C, Zhang Z, Lin Z, Hu J, Lu M, Wang Z, Qi Y, Zhang L, Pan R, Zhao Z, Lu L, Liao W and Lu X: AFP promotes HCC progression by suppressing the HuR-mediated Fas/FADD apoptotic pathway. Cell Death Dis 11(10): 822, 2020. PMID: 33009373. DOI: 10.1038/s41419-020-03030-7

45 Proctor MJ, Morrison DS, Talwar D, Balmer SM, Fletcher CD, O'Reilly DS, Foulis AK, Horgan PG and McMillan DC: A comparison of inflammation-based prognostic scores in patients with cancer. A Glasgow Inflammation Outcome Study. Eur J Cancer 47(17): 2633-2641, 2011. PMID: 21724383. DOI: $10.1016 /$ j.ejca. 2011.03 .028
46 Aino H, Sumie S, Niizeki T, Kuromatsu R, Tajiri N, Nakano M, Satani M, Yamada S, Okamura S, Shimose S, Sumie H, Torimura $\mathrm{T}$ and Sata M: Clinical characteristics and prognostic factors for advanced hepatocellular carcinoma with extrahepatic metastasis. Mol Clin Oncol 2(3): 393-398, 2014. PMID: 24772306. DOI: $10.3892 / \mathrm{mco} .2014 .259$

47 Ogawa E, Toyoda H, Iio E, Jun DW, Huang CF, Enomoto M, Hsu YC, Haga H, Iwane S, Wong G, Lee DH, Tada T, Liu CH, Chuang WL, Hayashi J, Cheung R, Yasuda S, Tseng CH, Takahashi H, Tran S, Yeo YH, Henry L, Barnett SD, Nomura H, Nakamuta M, Dai CY, Huang JF, Yang HI, Lee MH, Jun MJ, Kao JH, Eguchi Y, Ueno Y, Tamori A, Furusyo N, Yu ML, Tanaka Y, Nguyen MH and Real-World Evidence from the Asia Liver Consortium Investigators.: Hepatitis $\mathrm{C}$ virus cure rates are reduced in patients with active but not inactive hepatocellular carcinoma: A practice implication. Clin Infect Dis 71(11): 28402848, 2020. PMID: 31777940. DOI: 10.1093/cid/ciz1160

Received February 3, 2021

Revised February 18, 2021 Accepted February 19, 2021 